\title{
Abundance of stable ergodicity
}

\author{
Christian Bonatti, Carlos Matheus, Marcelo Viana and Amie Wilkinson
}

\begin{abstract}
We consider the set $\mathcal{P} \mathcal{H}_{\omega}(M)$ of volume preserving partially hyperbolic diffeomorphisms on a compact manifold having 1-dimensional center bundle. We show that the volume measure is ergodic, and even Bernoulli, for any $C^{2}$ diffeomorphism in an open and dense subset of $\mathcal{P H}_{\omega}(M)$. This solves a conjecture of Pugh and Shub, in this setting.
\end{abstract}

Mathematics Subject Classification (2000). 37D30

Keywords. Partial hyperbolicity, stable ergodicity, accessibility, Lyapunov exponents, nonuniform hyperbolicity.

To Charles and Mike: Happy 60th birthdays!

\section{History}

A fundamental problem, going back to Boltzmann and the foundation of the kinetic theory of gases, is to decide how frequently conservative dynamical systems are ergodic.

A first striking answer was provided by KAM (Kolmogorov, Arnold, Moser) theory: ergodicity is not a generic property, in fact there are open sets of conservative systems exhibiting positive volume sets consisting of invariant tori supporting minimal translations.

In sharp contrast with this elliptic type of behavior, ergodicity prevails at the other end of the spectrum, namely, among strongly hyperbolic systems. Indeed, after partial results of Hopf and Hedlund, Anosov proved that the geodesic flow of any compact manifold with negative curvature is ergodic. In fact, the same is true for any sufficiently smooth conservative uniformly hyperbolic flow or diffeomorphism.

By the mid-nineties, Pugh and Shub proposed to address the ergodicity problem in the context of partially hyperbolic systems, where the tangent space splits

This work was conceived during the Colloque M. Herman at the Institut H. Poincaré. MV was partially supported by the Convênio Brasil-França em Matemática and the Université de Bourgogne. CM was partially supported by Faperj. AW was partially supported by NSF Grant \#DMS-0100314 
into uniformly contracting (stable), uniformly expanding (unstable), and "neutral" (central) directions. To summarize their main theme:

\section{A little hyperbolicity goes a long way in guaranteeing ergodicity.}

In more precise terms, in [9] they proposed the following

Conjecture. Stable ergodicity is a dense property among $C^{2}$ volume preserving partially hyperbolic diffeomorphisms.

At about the same time, there was a renewed interest in the geometric and ergodic properties of partially hyperbolic systems in the broader context of possibly non-conservative dynamical systems. A main goal here was to establish existence and finiteness of SRB (Sinai, Ruelle, Bowen) measures, and to characterize their basins of attraction.

Thus the general theme of partially hyperbolic dynamics evolved into a very active research field, with contributions from a large number of mathematicians. See, for instance, $[2,5]$ for detailed accounts of much progress attained in the last few years.

\section{Result}

The purpose of this note is to point out that, putting together recent results by Shub, Wilkinson [10] followed by Baraviera, Bonatti [1], by Bonatti, Viana [3] followed by Burns, Dolgopyat, Pesin [4], and by Dolgopyat, Wilkinson [6], one obtains a proof of the conjecture stated above, when the central direction is 1dimensional.

Theorem. Let $M$ be a compact manifold endowed with a smooth volume form $\omega$, and $\mathcal{P} \mathcal{H}_{\omega}(M)$ be the set of all partially hyperbolic diffeomorphisms having 1dimensional center bundle and preserving the volume form.

Then the volume measure defined by $\omega$ is ergodic, and even Bernoulli, for any $C^{2}$ diffeomorphism in a $C^{1}$ open and dense subset of $\mathcal{P} \mathcal{H}_{\omega}(M)$.

The proof of the theorem follows. In fact, we prove a bit more: every $C^{2}$ diffeomorphism in $\mathcal{P} \mathcal{H}_{\omega}(M)$ is $C^{1}$ approximated by another $C^{2}$ diffeomorphism in $\mathcal{P H}_{\omega}(M)$ which is stably Bernoulli. Note that it is not known whether $C^{2}$ maps are dense in $\mathcal{P H}_{\omega}(M)$.

Throughout, all maps are assumed to be volume preserving. First, [1] extends the technique of [10], to prove that every partially hyperbolic diffeomorphism may be $C^{1}$ approximated by another for which the integrated sum of all Lyapunov exponents along the central direction is non-zero. Under our dimension assumption, this just means that the integrated central Lyapunov exponent is non-zero, for 
a $C^{1}$ open and dense subset $\mathcal{O}_{1}$ of partially hyperbolic diffeomorphisms. Let us decompose $\mathcal{O}_{1}$ as $\mathcal{O}_{-} \cup \mathcal{O}_{+}$, according to whether the integrated central exponent is negative or positive. Up to replacing $f$ by its inverse, we may suppose that $f \in \mathcal{O}_{-}$. For such $f$, there is a positive volume set of points with negative central Lyapunov exponent.

Next, also for $f$ in a $C^{1}$ open and dense subset $\mathcal{O}_{2}$, [6] proves that the diffeomorphism has the accessibility property: any two points may be joined by a path formed by finitely many segments contained in leaves of the strong-stable foliation or the strong-unstable foliation. In fact, they prove more: every $C^{2}$ diffeomorphism is $C^{1}$ approximated by other $C^{2}$ diffeomorphisms with this accessibility property.

We conclude that each $f \in \mathcal{O}_{ \pm} \cap \mathcal{O}_{2}$ is ergodic with respect to volume, by using Theorem 2 of [4], which builds on techniques of [3]: If a $C^{2}$ partially hyperbolic volume preserving diffeomorphism of a compact smooth Riemannian manifold is accessible and has negative central exponents on a set of positive measure, then it is ergodic and has negative central exponents almost everywhere.

Finally, the same arguments extend directly to any iterate $f^{n}, n \geq 1$. Indeed, $f^{n} \in \mathcal{O}_{ \pm}$if and only if $f \in \mathcal{O}_{ \pm}$, and $f^{n}$ is accessible if and only if $f$ is, since the two maps have the same strong foliations. This shows that $f^{n}$ is ergodic, for every $n \geq 1$, whenever $f \in \mathcal{O}_{ \pm} \cap \mathcal{O}_{2}$. Using Theorem 8.1 of Pesin [8], we conclude that $f$ is Bernoulli.

\section{Notation used}

To conclude, we give the technical definitions of the notions involved.

Let $M$ be a compact manifold endowed with a volume form $\omega$. A volume preserving diffeomorphism $f: M \rightarrow M$ is stably ergodic if the volume measure defined by $\omega$ is ergodic for any $C^{2}$ diffeomorphism in a $C^{1}$-neighborhood of $f$.

A diffeomorphism $f: M \rightarrow M$ is partially hyperbolic if there is a splitting $T M=E^{s} \oplus E^{c} \oplus E^{u}$ of the tangent bundle into three invariant bundles (with positive dimension) and there exists $m \geq 1$ such that

$$
\left\|D f^{m} \mid E^{s}\right\| \leq \frac{1}{2} \quad \text { and } \quad\left\|D f^{-m} \mid E^{u}\right\| \leq \frac{1}{2}
$$

and

$$
\left\|D f^{m} \mid E^{s}\right\|\left\|\left(D f^{m} \mid E^{c}\right)^{-1}\right\| \leq \frac{1}{2} \text { and }\left\|\left(D f^{m} \mid E^{u}\right)^{-1}\right\|\left\|D f^{m} \mid E^{c}\right\| \leq \frac{1}{2} .
$$

The first condition means that $E^{s}$ is uniformly contracting and $E^{u}$ is uniformly expanding. The last one means that the splitting is dominated. ${ }^{1}$

\footnotetext{
1 Because we compare expansion and contraction rates at the pointwise level, rather than uniformly over the whole manifold, our definition of partial hyperbolicity is more general than the definition in [4]. Nonetheless the main results in [4] extend in a fairly straightforward fashion to our setting.
} 
We denote $\mathcal{P} \mathcal{H}(M)$ the space of partially hyperbolic $C^{1}$ diffeomorphisms on $M$ with $\operatorname{dim} E^{c}=1$, and $\mathcal{P} \mathcal{H}_{\omega}(M)$ the subset of volume preserving diffeomorphisms.

Let $f \in \mathcal{P H}(M)$. Then the stable bundle $E^{s}$ and the unstable bundle $E^{u}$ are uniquely integrable. The corresponding integral foliations, respectively strongstable $\mathcal{F}^{s}$ and strong-unstable $\mathcal{F}^{u}$ are invariant, and their leaves are uniformly contracted by all forward and backward iterates of $f$, respectively.

We say that $f \in \mathcal{P} \mathcal{H}(M)$ has the accessibility property if any two points of $M$ may be joined by a path formed by finitely many segments contained in leaves of the strong-stable foliation or the strong-unstable foliation.

\section{Questions}

One would like to remove the assumption on the central dimension.

Another important open problem is the $C^{r}$ version of the conjecture, any $r>1$. In this direction, Niţică, Török [7] prove $C^{r}$ density of accessibility assuming a $r$ normally hyperbolic 1-dimensional, integrable central bundle with at least two compact leaves.

Here we prove ergodicity assuming $C^{2}$ regularity. While ergodic systems always form a $G_{\delta}$, it is not known whether $C^{2}$ maps are dense in the space $C^{1}$ volume preserving diffeomorphisms; see Zehnder [11]. So it remains open whether ergodicity is generic (dense $G_{\delta}$ ) among $C^{1}$ partially hyperbolic with 1-dimensional central bundle.

\section{References}

[1] A. Baraviera and C. Bonatti, Removing zero central Lyapunov exponents, Ergod. Th. Es Dynam. Sys. 23 (2003), 1655-1670.

[2] C. Bonatti, L. J. Díaz and M. Viana, Dynamics beyond uniform hyperbolicity: A global geometric and probabilistic perspective, Encyclopedia Math. Sciences, Springer-Verlag, 2004.

[3] C. Bonatti and M. Viana, SRB measures for partially hyperbolic systems whose central direction is mostly contracting, Israel J. Math. 115 (2000), 157-193.

[4] K. Burns, D. Dolgopyat and Ya. Pesin, Partial hyperbolicity, Lyapunov exponents and stable ergodicity, J. Statist. Phys. 108 (2002), 927-942. Dedicated to David Ruelle and Yasha Sinai on the occasion of their 65th birthdays.

[5] K. Burns, C. Pugh, M. Shub and A. Wilkinson, Recent results about stable ergodicity, in: Smooth ergodic theory and its applications (Seattle WA, 1999), volume 69 of Procs. Symp. Pure Math., pages 327-366. Amer. Math. Soc., 2001.

[6] D. Dolgopyat and A. Wilkinson, Stable accessibility is $C^{1}$ dense, Astérisque 287 (2003), $33-60$.

[7] V. Niţică and A. Török, An open dense set of stably ergodic diffeomorphisms in a neighborhood of a non-ergodic one, Topology 40 (2001), 259-278.

[8] Ya. B. Pesin, Characteristic Lyapunov exponents and smooth ergodic theory, Russian Math. Surveys 324 (1977), 55-114.

[9] C. Pugh and M. Shub, Stably ergodic dynamical systems and partial hyperbolicity, $J$. Complexity 13 (1997), 125-179. 
[10] M. Shub and A. Wilkinson, Pathological foliations and removable zero exponents, Invent. Math. 139 (2000), 495-508.

[11] E. Zehnder, Note on smoothing symplectic and volume preserving diffeomorphisms, Lect. Notes in Math. 597 (1977), 828-854.

\section{Christian Bonatti}

Université de Bourgogne

Laboratoire de Topologie

UMR $5584 \mathrm{du}$ CNRS

BP 47870

21078 Dijon Cedex

France

e-mail: bonatti@u-bourgogne.fr

Marcelo Viana

IMPA

Estrada D. Castorina 110

22460-320 Rio de Janeiro

Brazil

e-mail: viana@impa.br
Carlos Matheus

IMPA

Estrada D. Castorina 110

22460-320 Rio de Janeiro

Brazil

e-mail: matheus@impa.br

Amie Wilkinson

Department of Mathematics

Northwestern University

2033 Sheridan Road

Evanston, IL 60208-2730

USA

e-mail: wilkinso@math.northwestern.edu

(Received: February 28, 2003)

(1) To access this journal online:

(20) http://www.birkhauser.ch 